\title{
Kinematic analysis on the serve technique of world's elite tennis player Novak Djokovic
}

\author{
TaoYang ${ }^{1, a^{*}}$, Jihe Zhou ${ }^{2, b}$ and JunGuo ${ }^{3, c}$ \\ ${ }^{1}$ Chengdu sport University. Chengdu, Sichuan, China \\ ${ }^{2}$ Chengdu sport University. Chengdu, Sichuan, China \\ ${ }^{3}$ Chengdu sport University. Chengdu, Sichuan, China \\ a1219200579@qq.com, bjihezhou123@163.com ${ }^{\mathrm{c}}$ 328095703@qq.com
}

\begin{abstract}
Keywords: Three-dimensional photograph analytical method; Tennis; Kinematical analysis.
Abstract. Serve technique plays an important role in the tennis competition, and it's an important way to score. In virtue of three-dimensional photograph analytical method, this research presents a kinematical analysis on key links of serve technique by Novak Djokovic, the champion of men's singles in 2014 China Open, so as to obtain kinematical parameters of serve technique by the world excellent men's tennis player, reveal kinematics characteristics of serve technique and offer theoretical instructions to coaches and athletes for proper understanding and mastery of such a technique during training.
\end{abstract}

\section{Introduction}

Serve plays a vital role in the tennis match, giving full play to the serve power, and making a strong pressure on competitors so as to cloud their judgments and lower the quality of the serve, as a result, seizing the initiative and creating more opportunities to score in the competition. At the same time, the serve is also the only one tennis technique, which is controlled by the athletes themselves without the competitors' constraints. Therefore, whether a competition wins or not depends on the serve to a great extent. In the international tennis competition, many excellent athletes own the unique serve technique, which has a strong attack effect and fully reflects the strength, speed, unexpected drop placement and strong rotation variation. For example, the world champion Novak Djokovic, who is considered to have the most comprehensive serve technique, so it's worth further studying about the movement structure of the elite athletes, and seeking for the characteristics and laws in the tennis training practice. According to a study, Pang Yongkun ${ }^{[1]}$ has the following conclusions: the serve is the main mean for Novak Djokovic to scoring. Aiming at Novak Djokovic's tactical analysis in 2011-2013 in the Australian open final, Sun Lei makes use of the method of consulting literature, mathematical statistics, comparative analysis and video observation, finally, he shows that Novak Djokovic takes an advantage over than other competitions in the serve ${ }^{[2]}$, so it reveals the quality of serve is high and the placement is especially good. In a word, the biomechanical study about Novak Djokovic's serve technique has a great significance in theory and practice.

\section{Object and Method of Study}

Object. Research object is Novak Djokovic, Men's singles champion in 2014 China Open, who has won seven Grand Slam singles trophy by the end of 2014.

Method.three-dimensional photograph analysis, In 2014 China Open, it employed two JVC9800 cameras $(5 \mathrm{OfpS})$ in the fixed point form to shoot the entire serve process of Novak Djokovic from different angles with No.1 camera right behind the side line of the tennis court and No.2 camera right in front of the base line, showing an included angle of about $45^{\circ}$ between main optical axes of the two cameras .(the position of two cameras is shown in Fig. 1) Star high titanium 3D Signal TEC V3.2HDC analysis system developed by Beijing Senmiaoxin Science and Technological Development Company is adopted to analyze the video shot picture by picture, with original data obtained smoothened by low-pass digital filtering method and a truncation frequency of 8 hertz. The human body model hereby adopts Dempster model (16 links and 22 artis parameters). Another three measurement points such as 
racket head, shoulder-hip projected angles and ball are added during analysis as required by this research. According to the characteristics of tennis serve, upper data mainly selected from elbow kinematics data of hander arm and holding arm and lower data mainly selected from knee kinematics data of the two supporting leg were used to analyze and illustrate.

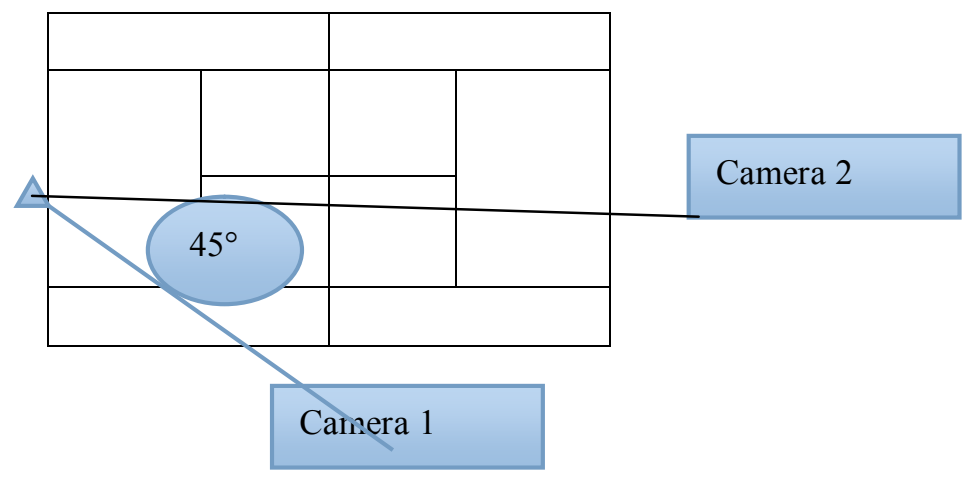

The position of two cameras (Fig. 1)

\section{Result and Discussion}

Stage division of Serve.Novak Djokovic is the Champion in Chinese Open Tennis, who uses the FB technique in the serve position, considering about the research purpose and results and analysis demands, this paper divides the action of tennis serve technique into four stages as follows.

T1: the ball at the lowest point.

T2: the moment of the ball away from the hand

T3: the moment of the ball reached the highest point

T4: The maximum moment of the knee flexion

T5: : the end of the backward swing stage

T6: the moment of stroke

T7: the landing moment of left foot

T1-T4: Toss ball and lift racket stage: from the ball in the lowest point to the maximum moment of the knee flexion.

T4-T5: backward swing stage: from the maximum moment of the knee flexion to the end of the backward swing stage.

T5-T6: forward swing stage: from the end moment of the backward swing stage to the moment of stroke.

T6-T7: landing buffer stage: from the moment of stroke to the landing moment of left foot.

Serve related data interpretation. Hip joint angle: the angle between the ipsilateral shoulder and knee joint at the hip place.

Knee joint angle: the angle between the ipsilateral hip and ankle knee joint premises in the knee joint

Elbow joint angle: the angle between the ipsilateral shoulder joint and wrist joint in elbow joint.

Shoulder joint angle: the angle between the ipsilateral hip and elbow joint in the shoulder joint.

Shoulder-hip projected angles: the angle of double shoulder and hip connection line in plane 3 (X-Z).

Research result and analysis at the stage of tossing ball and lifting racket. Toss ball and lift racket,the stage means the process from the moment of the ball at the lowest point to the moment of maximum knee flexion. The purpose of this stage is tossing the ball firmly into the fixed position. The action of the stage mainly analyzes and discusses about the height of the ball away from hand, the angle of elbow joint and knee joint, and other several aspects. At this stage, the higher height of the ball away from hand, the longer distance controlled by hand, which can strengthen the hand to control the ball and make the route of the ball thrown to the air relatively stable. The height of the ball away from the hand is too low, on the one hand, it is not conducive to acceleration of the ball, on the other hand, is not conducive to controlling the movement direction of tossing the ball, the height of the ball away from 
hand is $1.95 \mathrm{~m}$ and the ratio of the height with Novak Djokovic's height is 1.04 . According to the data of elite tennis players: the height of the ball away from the hand is $2.02 \mathrm{~m}$ from Enquist ${ }^{[3]}$, then compared with this, the height is more suitable from Novak Djokovic. However, the buckling of the toss arm is one of the most important factors deciding the height of the ball away from the hand. Lower height will have an adverse impact on the quality of tossing the ball, so it is the most favorable for serve when the toss arm stretches straightly. The elbow joint extension amplitudes of Novak Djokovic's toss arm are about $174.6^{\circ}$. the elbow joint angle varies smaller, the shoulder joint angles of Novak Djokovic's toss arm and elbow joint angles are about $171.8^{\circ}$ and $116.4^{\circ}$ respectively, these data suggest that athlete's toss arm stretches quite straight, It is conducive to tossing the ball better. The angle of knee joints can affect the amplitude of movement of the next stage, when the angle of bending knee is smaller, it can produce effective thrust force. The flexion angle of Novak Djokovic's left knee joint is $110.3^{\circ}$ at the maximal knee flexion moment. It's reported that excellent occupation athletes' knee joint angle of the serve hand's opposite direction is about $100^{\circ}$, when the center of body weight is down to the lowest point. Apparently, Novak Djokovic's minimum angle of knee joint is in a reasonable range at this moment. Novak Djokovic's right knee joint angle is $150.6^{\circ}$, when the ball reached to the highest point. At this stage, the maximum height of tossing ball reaches $3.45 \mathrm{~m}$, these data suggest that the bending angle of Novak Djokovic's knee joint is very large and the height of the ball reached to the highest point is very high, so it is fully prepared for the knee stretching rapidly. Novak Djokovic's shoulder and hip Link projection angle is about $38.65^{\circ}$, at this stage, it indicates that the torsion of athlete body is great, which will increase the torsional power for swinging towards the direction of stroke.

Research results and analysis of the backward swing stage.Backward swing stage, the stage means the process from the maximum moment of the knee flexion to the end of the backward swing stage. In this stage, the purpose is to make the athletes come into being "arched body" posture and form the action of "scratch back", Thereby it laid a solid foundation for swinging forward. The greater the knee range of motion, the faster movement speed, which can create favorable conditions for the follow-up action. The stretch amplitudes of Novak Djokovic's left knee joint is about $75^{\circ}$, the average angular speed of knee's pedal and stretch is $210.6 \%$, indicating that the stretching and movement speed of Novak Djokovic's knee is very quick, With a strong ground force makes the center of body weight rises rapidly, so that the racquet descended because of inertia, thereby increasing the working distance of swinging. At the same time, keeping the larger angle of elbow joint is propitious to the rapid stretch action of elbow to swing forward, improve the speed of elbow stretch. Novak Djokovic ,the minimum of the right elbow joint flexion angle is $72.5^{\circ}$, and this shows that Novak Djokovic's elbow flexion such that the movement direction of the racket relative to the body is downward, the amplitude of swing is larger, it makes the swing distance longer, and at the same time, making fully preparation for forward swing. The research shows that: the larger shoulder joint angle can not only increase the range of "scratch back", but also make the elastic potential energy store more, at the end of the backward swing, the angle of Novak Djokovic's right shoulder joint is $78.6^{\circ}$, PeteSampras and Thomas Enqvist's shoulder joint angles are $80.98^{\circ}, 74.82^{\circ}$ respectively ${ }^{[3]}$, compared with these excellent athletes, Novak Djokovic's shoulder joint angle is relatively large. At the end of the backward swing stage, Novak Djokovic's left hip joint angle is $161.7^{\circ}$, the right hip angle of $141.0^{\circ}$, the left knee joint angle of $174.8^{\circ}$, the right knee angle of $165.1^{\circ}$, this shows that Novak Djokovic's knee joint and hip joint has fully extended, making his body come into being "arched body" posture and form a "scratch back" action, at the moment, the racket has been away from the contact point, and it appears behind the athlete's body.

Research results and analysis of the forward swing stage.Forward swing stage, the stage means the process from the end of the forward swing stage to the moment the stroke. Purpose of this stage is to make the racket obtain greater movement speed, and then make the ball firmly to shoot to competitor's serving field. The higher the height of stroke is, the greater the possibility of hitting the ball over the net is, while the height of stroke is too low , will not only increase the technical difficulty of the action of stroke, but also make the ball easily come into the net. The height of stroke is $2.85 \mathrm{~m}$ 
(1.52 times of his body height). The research shows that the stroke height of these international excellent athletes is about 1.50 times of the body height. According to comparison, Novak Djokovic's stroke height is reasonable. The drop between the height of tossing the ball to the highest point and the height of the stroke point is about $0.40 \mathrm{~m}$, studies have shown that the above drop should be about $50 \mathrm{~cm}$, and some even is in the range of $20 \mathrm{~cm}$, thus it can be seen, Novak Djokovic's drop is within a reasonable range. The smaller horizontal distance is conducive to delivering the force of lower limbs, at this stage, the horizontal distance between the stroke point and the point of tossing the ball is $0.65 \mathrm{~m}$. This shows that smaller horizontal distance is conducive to delivering the strength of Novak Djokovic's legs. At the moment of the stroke, Keep the shoulder joint angle and elbow joint angle large, then it can increase rotating radius in distal part around the shoulder joint, and it is conducive to the completion of the "whiplash" action, thereby increasing the ball speed. at the moment of stroke, Novak Djokovic's left hip angle of $167.1^{\circ}$, the right elbow joint angle of $169.6^{\circ}$, right shoulder angle of $156.6^{\circ}$, this shows that Novak Djokovic's grip arm has fully stretched, slightly bent, the body has fully stretched, the center of body weight has moved to the front. The tennis serve action belongs to the " whiplash " action, so the reasonable rhythm of action should be that the speed from the lower joint to the upper joint increases gradually, the greater the increment of each links speed are, the better the transmission effect of the " whiplash " action is' At the moment of stroke, the speeds of Novak Djokovic's shoulder joint, elbow joint, wrist joint, the racquet head were $3.42 \mathrm{~m} / \mathrm{s}, 3.76 \mathrm{~m} / \mathrm{s}, 6.24 \mathrm{~m} / \mathrm{s}, 24.35 \mathrm{~m} / \mathrm{s}$, respectively, so it indicated that Novak. Djokovic's serve is in accord with the principles of whiplash action.

Research results and analysis of the landing buffer stage.Landing buffer stage, the stage means the process from the moment of stroke to the left foot land and the Purpose of this stage is to keep body balance and make a full preparation for the next movement. At the stage, after the ball hitting the racket, the racket is on one side of the body, then stops on the other side, the racket head presses downward quickly, left foot lands on the ground, the right shoulder fully extends forward, the right leg extends backward, the right arm holds the abdomen so as to maintain the body balance and the center of body gravity follows up the next movement. At the left foot landing on the ground, Novak Djokovic's right knee extends backward and his knee joint angle is $165.0^{\circ}$. This shows that the center of his gravity has been completely being in the front, in order to maintain body balance, the right knee joint stretches backward. At this stage, Novak Djokovic's shoulders rotate largely, and the angle of his shoulders rotating is $183^{\circ}$ at the moment from tossing ball to left foot landing on the ground, so it shows that Novak Djokovic's shoulders rotate more fully and it will be conducive to the rotation of body and increasing the speed of racquet head.

\section{Conclusion}

Through above analysis and discussion about the world champion Novak Djokovic's serve technique, it is worth learning for us about Novak Djokovic's serve technique model. The main indicators of the overall action are as follow:

Toss ball and lift racket stage.The height of the ball away from hand is higher from Novak Djokovic, it is $1.95 \mathrm{~m}$, and the ratio of the height with his height is 1.04 . In the entire process of tossing the ball, which is from the ball at the lowest point to the ball away from the hand, the elbow joint extension amplitudes of Novak Djokovic's toss arm is about $174.6^{\circ}$, at the moment of the ball away from hand, the shoulder joint angle of Novak Djokovic's toss arm is about $116.4^{\circ}$, the angle of elbow joint is about $171.8^{\circ}$, the elbow joint of Novak Djokovic's toss arm stretched quite straight, it was conducive to controlling the ball, and making the route of throwing the ball to the air quite stable. The maximum height of tossing ball reaches $3.45 \mathrm{~m}$, the flexion angle of Novak Djokovic's left knee joint is $110.3^{\circ}$.at the maximal knee flexion moment, it is fully prepared for the rapid stretching of Novak Djokovic's knees, this shows that in the condition of leg strength allowing, increasing the depth of the knee flexion is beneficial to the subsequent serve action. Novak Djokovic's shoulder and hip Link projection angle is about $38.65^{\circ}$ at this stage, Novak Djokovic's body has a great torsion, which increases the torsional strength for swinging towards the direction of stroke. 
Backward swing stage, the stretch amplitude of Novak Djokovic's left knee joint is about $75^{\circ}$, the average angular speed of knee's pedal extension is $210.6^{\circ} / \mathrm{s}$, the extension and movement speed of Novak Djokovic's knee is very quick, With a strong ground making the center of body weight rise rapidly so that the racquet relative to athlete's back descends because of inertia, thereby increasing the working distance of swinging racket. The minimum flexion angle of the right elbow joint is $72.5^{\circ}$, Novak Djokovic's elbow has a flexion such that the movement direction of the racket relative to body is downward, the amplitude of swinging is larger, which makes the swing distance long, and at the same time, making a full preparation for swinging forward. The angle of Novak Djokovic's right shoulder joint is $78.6^{\circ}$, it can not only increase the range of "scratch back" but also make elastic potential energy store more. At the end of the backward swing stage, Novak Djokovic's left hip joint angle of $161.7^{\circ}$, the right hip angle of $141.0^{\circ}$, the left knee joint angle of $174.8^{\circ}$, the right knee angle of $165.1^{\circ}$, Novak Djokovic's knee joint and hip joint have a full stretch, which makes his body come into being "arched body" posture and form a "scratch back" movement at the moment. The racket has been away from the contact point, and it appears behind the athlete's body.

Forward swing stage,the height of stroke is $2.85 \mathrm{~m}$ (1.52 times of his body height). Novak Djokovic's stroke height is reasonable, the drop between the height of tossing ball to the highest point and the height of the stroke point is about $0.40 \mathrm{~m}$, the above drop is within a reasonable range, the horizontal distance between the stroke point and the point of tossing ball is $0.65 \mathrm{~m}$, it is conducive to delivering the strength of Novak Djokovic's legs. The right elbow joint angle of $169.6^{\circ}$, right shoulder angle of $156.6^{\circ}$, Novak Djokovic's grip arm is fully stretched, slightly bent, it can increase rotation radius in distal part around the shoulder joint, and it is conducive to the completion of Novak Djokovic's the "whiplash" action, thereby increasing the ball speed. At the moment of stroke, the speed of Novak Djokovic's shoulder joint, elbow joint, wrist joint, the racquet head was $3.42 \mathrm{~m} / \mathrm{s}, 3.76 \mathrm{~m} / \mathrm{s}$, $6.24 \mathrm{~m} / \mathrm{s}, 24.35 \mathrm{~m} / \mathrm{s}$, respectively, Novak. Djokovic's serve is in accord with the principles of whiplash action.

Landing buffer stage, at the landing moment of left foot, Novak Djokovic's right knee joint is stretching backward, the knee joint angle of $165.0^{\circ}$,Novak Djokovic's center of body weight has stayed completely in the front, the purpose of the action is to maintain the body balance.

\section{References}

[1] Yongkun Pang, The application and analysis of Djokovic's technique and tactics in 2011 four Grand Slam finals, Chengdu Sport University, 2013.

[2] Lei Sun, Analysis of Djokovic's technique and tactics in the Australian Open final of 2011-2013 in D, Taiyuan University of Technology, 2014.

[3] Yirong Li,Jihe Zhou; Kinematics analysis on serve technique of Thomas Enqvist and Carlos Moya; the Sixteenth National Symposium on biomechanics in sports (CABS 2013) in C. 2013.

[4] KaiHu; YunWang, Analysis on serve technique of excellent male tennis players Djokovic and Nadal's serve technique in the 2012 Australian Open final as an example ,J. School of physical education of Jiangxi Normal University, 2013.

[5] Junbo Lu, Biomechanical analysis on the serve technique of world elite male tennis players, D; Chengdu Sport University,2011. 